\title{
Nonlinear analysis of a simple model of temperature evolution in a satellite
}

\author{
Jose Gaite, Angel Sanz-Andres and Isabel Perez-Grande
}

\begin{abstract}
We analyse a simple model of the heat transfer to and from a small satellite orbiting round a solar system planet. Our approach considers the satellite isothermal, with external heat input from the environment and from internal energy dissipation, and output to the environment as black-body radiation. The resulting nonlinear ordinary differential equation for the satellite's temperature is analysed by qualitative, perturbation and numerical methods, which show that the temperature approaches a periodic pattern (attracting limit cycle). This approach can occur in two ways, according to the values of the parameters: (i) a slow decay towards the limit cycle over a time longer than the period, or (ii) a fast decay towards the limit cycle over a time shorter than the period. In the first case, an exactly soluble average equation is valid. We discuss the consequences of our model for the thermal stability of satellites.
\end{abstract}

\section{Introduction}

The design process of any spacecraft necessarily includes a thermal analysis, performed to guarantee that both the bus and the payload are kept along the whole mission within the appropriate temperature range [1]. The thermal environment of a satellite in orbit is very harsh, dominated by vacuum conditions which make difficult to find a way of cooling down the components of the spacecraft. Every single element of the satellite has to be thermally analysed to verify that its requirements are fulfilled. To do it, it is necessary a rather detailed design of the spacecraft, as it implies to calculate thermal loads and couplings between elements. This is usually carried out by commercial software tools based on numerical methods.

During the generic trade-off study, early in the design process, that is, when the concept of the spacecraft is still open, it is very convenient to assess the mean temperature of the satellite and its variation by means of analytical tools. This method provides valuable 
information about the feasibility of the spacecraft from the thermal point of view as well as the possibility of easily carrying out parametric analyses to study the influence of the different variables involved in the problem.

The purpose of the present study is to analyse the thermal behaviour of a small compact satellite spinning in a low orbit round a solar system planet (the Earth, say) as a function of time, using analytical or semi-analytical tools. In orbit, the spacecraft is subjected to periodic heat loads and it is expected to reach a thermally periodic state. Apart from obtaining the temperature oscillations once reached this periodic state, this analytical approach allows us to study the convergence of the satellite temperature to the periodic behaviour starting from different initial conditions, as it can occur from launch conditions or orbital manoeuvres.

The results are obtained by applying the energy balance equation to the satellite, taking into account both the external heat loads, i.e. the solar irradiation, the planet albedo (solar irradiation reflected on the planet) and the infrared (IR) radiation from the planet, as well as the internal dissipation.

We begin with the formulation of the energy balance equation as a non-dimensional ordinary differential equation (ODE), which is nonlinear and non-autonomous. Then, we consider an autonomous ODE that plays the role of an average of the actual equation. That autonomous equation is exactly soluble and guides our intuition of the behaviour of the actual equation and helps in its analysis. This analysis is carried out with qualitative, perturbation and numerical methods. Finally, we present our conclusions, regarding the design of satellites.

\section{Nonlinear ODE for the satellite temperature}

Let us assume that the satellite is approximately isothermal, so we characterize it by its temperature $T$. The heat input consists of an external flow coming from the space environment, in particular, the solar irradiation, the planetary albedo and the planetary IR radiation, and the internal heat due to the equipment dissipation. The solar irradiation and the planetary albedo have periodic variation, according to the position of the satellite on its orbit. On the contrary, the planetary IR radiation and the internal heat operate at constant rate.

Let us assume that the solar irradiation changes from constant to vanishing (when the satellite is in the planet's shade), whereas the albedo depends on the angle between the sunlight and the vertical to the satellite (and is much smaller in absolute value). Furthermore, we assume that the albedo vanishes for one fraction of a period whereas the satellite's night (when it is in the planet's shade) lasts for another (smaller) fraction. We denote the heat rate due to solar irradiation by $\dot{Q}_{s}$, and the maximum albedo heat rate by $\dot{Q}_{a}$ (at the satellite's noon). In addition, let the constant rate source have power $\dot{Q}_{c}$ (the sum of the equipment dissipation and planetary IR radiation). Then, the energy balance equation that yields the temperature $T$ is

$$
C \dot{T}(t)=\dot{Q}_{s} f_{s}(\nu t)+\dot{Q}_{a} f_{a}(\nu t)+\dot{Q}_{c}-A \epsilon \sigma T(t)^{4}
$$




$$
\begin{array}{r}
f_{s}(x)=1,0 \leq x \leq x_{1} \text { or } 1-x_{1} \leq x \leq 1 ; f_{s}(x)=0, x_{1}<x<1-x_{1} \\
f_{a}(x)=\cos (2 \pi x), 0 \leq x \leq x_{2} \text { or } 1-x_{2} \leq x \leq 1 ; f_{a}(x)=0, x_{2} \leq x \leq 1-x_{2} \\
f_{s, a}(x)=f_{s, a}(x-1), x \geq 1
\end{array}
$$

Here $C$ is the satellite's thermal capacity, $\nu$ the orbital frequency, $A$ is the satellite's surface area, $\epsilon$ its emissivity, and $\sigma$ the Stefan-Boltzmann constant. The values of $x_{1}$ and $x_{2}$ are smaller than one half and they determine the fractions of the period with vanishing albedo or sunshine, respectively. In principle, $x_{2}=1 / 4$ and $x_{1}>x_{2}$ (we assume that the albedo vanishes for half a period whereas the satellite's night is shorter: we take it as one fifth of the period, that is, $x_{1}=2 / 5$, in an example below).

We can write this equation in non-dimensional form by defining $a=A \epsilon \sigma /(C \nu), k_{s}=$ $a^{1 / 3} \dot{Q}_{s} /(C \nu), k_{a}=a^{1 / 3} \dot{Q}_{a} /(C \nu), k_{c}=a^{1 / 3} \dot{Q}_{c} /(C \nu)$, and non-dimensional temperature variable $\theta=a^{1 / 3} T$ and time variable $\nu t$ (which we still denote $t$ for notational simplicity). Then,

$$
\dot{\theta}(t)=k_{c}+k_{s} f_{s}(t)+k_{a} f_{a}(t)-\theta(t)^{4} .
$$

Unfortunately, this nonlinear ODE cannot be reduced to a quadrature; but we can deduce its relevant properties, nevertheless.

We note that, if we remove the oscillating terms, the resulting equation

$$
\dot{\theta}(t)=k-\theta(t)^{4}
$$

(where $k=k_{c}$ ) is immediately reduced to a quadrature, which can be integrated analytically. Moreover, we can deduce the qualitative behaviour of the solutions in a straightforward way: there is one fixed point, $\theta_{\text {eq }}=k^{1 / 4}$, and it is stable. It is the equilibrium temperature, at which the heat input and the radiation output balance one another. When the temperature is close to it, $\theta=k^{1 / 4}+\Delta \theta$, we obtain the linear ODE

$$
\dot{\Delta} \theta(t)=-4 k^{3 / 4} \Delta \theta(t)
$$

with solution

$$
\Delta \theta(t)=\Delta \theta(0) \exp \left(-4 k^{3 / 4} t\right)
$$

Therefore, $\Delta \theta$ halves in a time $\Delta t=(\ln 2 / 4) k^{-3 / 4}=0.17 / \theta_{\mathrm{eq}}{ }^{3}$; and the larger is $\theta_{\text {eq }}$, the shorter it takes to reach equilibrium. We have checked that the exact solution of Eq. (2) is well approximated by the linear equation solution (3).

We remark that Eq. (2) can be naturally connected with Eq. (1) if we assume that $k$ is the average of $k_{c}+k_{s} f_{s}(t)+k_{a} f_{a}(t)$ over one period, namely, $k=k_{c}+2 x_{1} k_{s}+k_{a} / \pi$ (rather than $k=k_{c}$ ). We explore the consequences of this connection in the next section.

With $k=k_{c}+2 x_{1} k_{s}+k_{a} / \pi$, reverting to physical variables, we have

$$
T_{\mathrm{eq}}=\left[\frac{\dot{Q}_{c}+2 x_{1} \dot{Q}_{s}+\dot{Q}_{a} / \pi}{A \epsilon \sigma}\right]^{1 / 4} .
$$

Note that it does not depend on the parameters $C$ and $\nu$, associated to the time derivative of the temperature in the ODE in physical variables. 


\section{Solution of the nonlinear ODE for the temperature evolution}

In this section, we first present a qualitative analysis, which allows us to prove the existence of an attracting limit cycle. To obtain the transient behaviour and the properties of the limit cycle, we employ perturbation theory. Finally, we perform a numerical analysis of the ODE, to check the results of the preceding methods and to obtain concrete and more precise numerical results.

\subsection{Qualitative analysis}

Equation (1) is non-autonomous and therefore equivalent to an autonomous system of two ODE's, namely, the system formed by Eq. (1) and the trivial equation $\dot{t}=1$. The generic behaviour of an autonomous system of two first-order ODE's is to have "simple" attracting sets, namely, equilibrium points or limit cycles, because of the Poincaré-Bendixson theorem $[2,3,4]$. Given the nature of our problem, we can also apply the theory of ODE's with periodic coefficients (see, e.g., Ref. [5]): we can reduce the dynamics in the $(t, \theta)$-plane to the cylinder $[0,1) \times(0, \infty)$. Furthermore, we can consider $\theta$ as the radial coordinate and $2 \pi t$ as the angular coordinate of a plane with the origin excluded (equivalent to the mentioned cylinder). Given that there cannot be fixed points (since the equation $\dot{t}=1$ forbids it), the Poincaré-Bendixson theorem implies that the only possible attractors are limit cycles, with the period imposed by the heat input.

The Poincaré-Bendixson theorem states that a curve solution of a two-dimensional autonomous ODE that has no singularities (e.g., fixed points) and is contained in a compact domain for all $t \geq 0$ approaches a limit cycle. We use a consequence of this theorem: given two concentric closed curves limiting an annular region that is free of singularities and such that the vector of derivatives on the two curves points towards the inside of the region, there exists a limit cycle in this annulus.

To prove the uniqueness of the limit cycle, we appeal to Dulac's criterion for an annular region: if the autonomous system

$$
\dot{x}=P(x, y), \dot{y}=Q(x, y)
$$

is such that the divergence $\partial_{x} P+\partial_{y} Q$ has constant sign in an annular region, then this region contains at most one limit cycle. The proof of this criterion follows from Green's theorem [3].

It is easy to check that Eq. (1) is such that $\theta<\theta_{\min }=k_{c}{ }^{1 / 4} \Rightarrow \dot{\theta}>0$ and $\theta>\theta_{\max }=$ $\left(k_{s}+k_{a}+k_{c}\right)^{1 / 4} \Rightarrow \dot{\theta}<0$, for all $t$. Note that $\theta_{\min }$ and $\theta_{\max }$ correspond to the equilibrium temperatures with constant minimum or maximum heat input, respectively. Therefore, the trajectories in the plane with $\theta$ as the radial coordinate and $2 \pi t$ as the angular coordinate which begin inside the annulus defined by those two temperatures are confined in it, and there is (at least) one limit cycle (with an oscillation in $\theta$ confined to take place within those 
values). Furthermore, this limit cycle is unique, because $\operatorname{div}\left(k_{c}+k_{s} f_{s}(t)+k_{a} f_{a}(t)-\theta^{4}, 1\right)=$ $-4 \theta^{3}<0$.

The trajectories inside the annulus are alternately increasing and decreasing, as the right-hand side of Eq. (1) changes sign. To be precise, if we define the periodic function

$$
\theta_{\lim }(t)=\left[k_{c}+k_{s} f_{s}(t)+k_{a} f_{a}(t)\right]^{1 / 4},
$$

with minimum and maximum values $\theta_{\min }$ and $\theta_{\max }$, respectively, the sign of $\dot{\theta}(t)$ changes when the trajectories cross it. This is represented in Fig. 1.

Using $\theta$ as the radial coordinate and $2 \pi t$ as the angular coordinate, Fig. 1 becomes the polar plot in Fig. 2. This polar plot is more useful to represent convergence to the limit cycle in a standard way.

We are interested in two questions: (i) finding the features of the limit cycle and the rate of convergence to it; (ii) analysing how this limit cycle and the rate of convergence depend on the constants $k_{c}, k_{s}, k_{a}$. These questions cannot be answered by a qualitative analysis, so we turn to other methods.

However, let us note that some more qualitative information can be obtained from a comparison with the non-oscillating Eq. (2): if $\theta_{\mathrm{eq}}=k^{1 / 4}=\left(k_{c}+4 k_{s} / 5+k_{a} / \pi\right)^{1 / 4}$ is sensibly smaller than one, the convergence time $\Delta t=\theta_{\mathrm{eq}}{ }^{-3}$ is large, so that it is consistent to consider Eq. (2) as an averaged equation. To be precise, Eq. (2) can be derived as an equation for the mean temperature in Eq. (1) by averaging it over one period. Therefore, the evolution is given by a long-time decay to $\theta_{\text {eq }}$ and a short-time oscillation about that long-time behaviour (as in Fig. 1).

Thus, it seems convenient to consider Eq. (1) as a perturbation of Eq. (2), namely, to consider the time-dependent (oscillating) functions $f_{a, s}$ as a perturbation. Then, we can employ standard perturbation methods [6]. We do so in the next section.

\subsection{Perturbation theory}

As we have explained above, our intention is to take advantage of the simple solution of Eq. (2) by taking it as the basis of a perturbation scheme. Therefore, we write Eq. (1) as

$$
\dot{\theta}(t)=k+\varepsilon f(t)-\theta(t)^{4},
$$

where $k=k_{c}+2 x_{1} k_{s}+k_{a} / \pi, f(t)=k_{c}-k+k_{s} f_{s}(t)+k_{a} f_{a}(t)$, and $\varepsilon$ is a formal perturbation parameter to be set to one at the end. Note that $f(t)$ is defined such that it has vanishing average over a period and, therefore, it represents the deviations about the mean value $k$. Furthermore, the solution of Eq. (5) must fulfill the initial condition $\theta(0)=\theta_{\text {in }}$. We assume an expansion of $\theta(t)$ of the form

$$
\theta(t)=\sum_{n=0}^{\infty} \varepsilon^{n} \theta_{n}(t)
$$



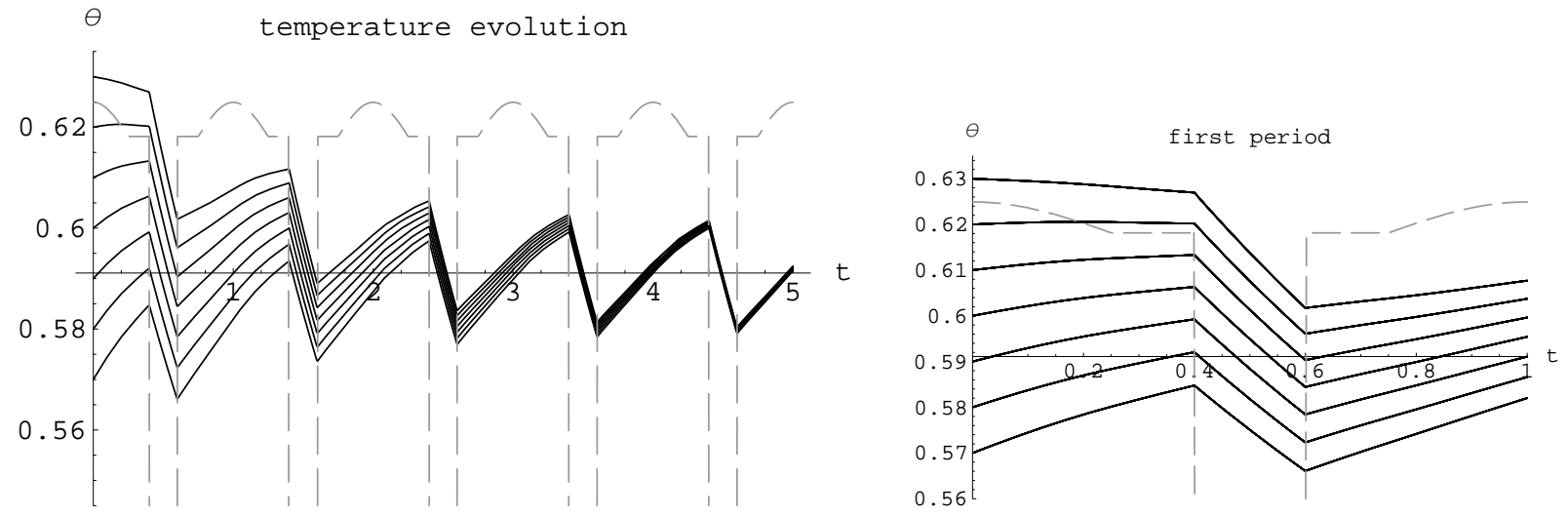

Figure 1: Results of the numerical analysis of the ODE with $k_{s}=0.13, k_{a}=0.007$ and $k_{c}=0.016$, explained in the text: (left) convergence to the attractor; (right) detail of the first period. Note how the trajectories change from increasing to decreasing, and viceversa, as they cross the dashed line $\theta_{\lim }(t)$ (the bottom of this line, at $k_{c}{ }^{1 / 4}=0.36$, is below the plot regions).

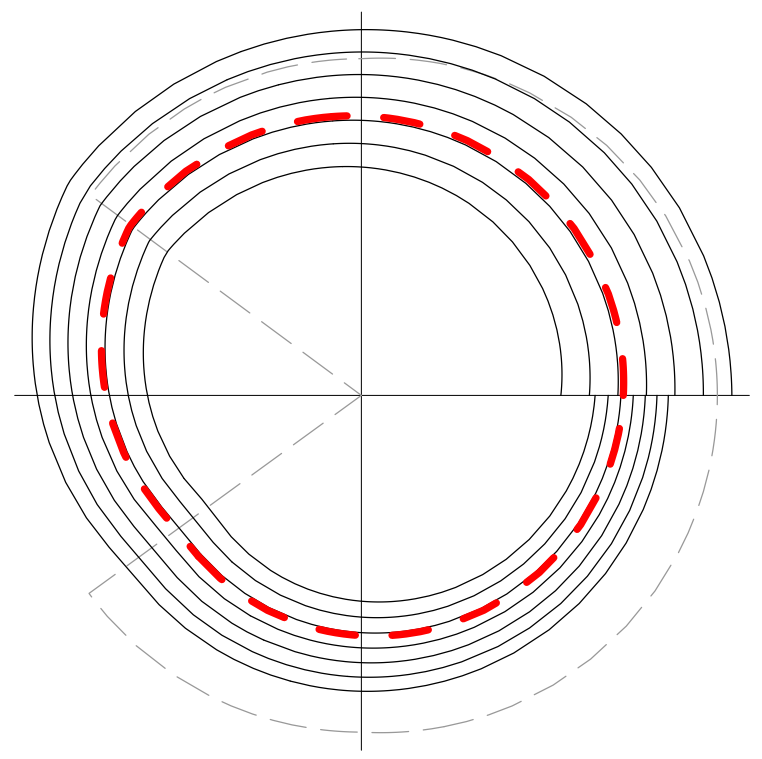

Figure 2: Polar plot of the trajectories in Fig. 1 (right): the radius is $\theta-0.5$ (subtracted to display well-separated lines) and the angle is $2 \pi t$. The limit cycle is the bold dashed line. 
and substitute it into Eq. (5). Equating to zero the successive powers of $\varepsilon$, we obtain the basis equation and an infinite set of linear ODE's, namely,

$$
\begin{array}{r}
\dot{\theta}_{0}(t)=k-\theta_{0}(t)^{4}, \\
\dot{\theta}_{1}(t)=f(t)-4 \theta_{0}(t)^{3} \theta_{1}(t), \\
\dot{\theta}_{2}(t)=-6 \theta_{0}(t)^{2} \theta_{1}(t)^{2}-4 \theta_{0}(t)^{3} \theta_{2}(t),
\end{array}
$$

The initial condition for $\theta_{0}(t)$ is $\theta_{0}(0)=\theta_{\text {in }}$, while the remaining equations fulfill $\theta_{n}(0)=0$.

These ODE's can be solved subsequently, namely, we can solve first the equation for $\theta_{0}(t)$ and, then, we can solve the equation for $\theta_{1}(t)$ and so onwards. Of course, the equation for $\theta_{0}(t)$ is Eq. (2), which we have already solved. The following equations are just firstorder linear inhomogeneous equations, which are soluble by quadratures [6]. However, their solution involves complicated integrals that cannot be made analytically. In particular, Eq. (7) has the solution

$$
\theta_{1}(t)=\frac{1}{I(t)} \int_{0}^{t} f(\tau) I(\tau) d \tau, \quad I(t)=\exp \left[4 \int_{0}^{t} \theta_{0}(\tau)^{3} d \tau\right]
$$

but $I(t)$ has no analytic expression.

To overcome this difficulty and obtain analytic expressions, we can use instead of the exact function $\theta_{0}(t)$ the approximated function given by Eq. (3), namely, $\theta_{0}(t)=\theta_{\text {eq }}+$ $\left(\theta_{\text {in }}-\theta_{\text {eq }}\right) \exp \left(-4 k^{3 / 4} t\right)$. Thus, the integral in $I(t)$ becomes a sum of integrals of exponential functions, which yield exponential functions again. In the large- $t$ limit (when $t \gg \theta_{\mathrm{eq}}{ }^{3}$ ), the exponentials decay and the value of $I(t)$ further simplifies to $I(t)=\exp \left(4 k^{3 / 4} t\right)$. Then,

$$
\theta_{1}(t)=\int_{0}^{t} f(\tau) \exp \left[-4 k^{3 / 4}(t-\tau)\right] d \tau=\int_{0}^{t} f(t-\tau) \exp \left[-4 k^{3 / 4} \tau\right] d \tau
$$

Furthermore, in the large- $t$ limit and given that $f(t)$ is periodic, we can extend the upper integration limit in the latter integral from $t$ to $\infty$. Thus, we have a periodic function, namely, an approximation to the limit cycle (past the transient regime). We can obtain a general expression of this periodic function through Fourier analysis.

\subsubsection{Fourier analysis}

Let us expand $f(t)$ in Fourier modes as

$$
f(t)=\sum_{m=-\infty}^{\infty} f_{m} e^{2 \pi i m t} .
$$

Since $f(t)$ is real and we assume that it is symmetric with respect to $t=0$, the coefficients $f_{m}$ are real and $f_{-m}=f_{m}$; in addition, $f_{0}=\int_{0}^{1} f(t) d t=0$. The other Fourier coefficients are given by

$$
f_{m}=\int_{0}^{1} f(t) e^{-2 \pi i m t} d t .
$$


It is straightforward to solve for $\theta_{1}(t)$ :

$$
\theta_{1}(t)=\sum_{m=-\infty}^{\infty} \frac{f_{m} e^{2 \pi i m t}}{2 \pi i m+4 k^{3 / 4}}=2 \sum_{m=1}^{\infty} f_{m} \frac{4 k^{3 / 4} \cos (2 \pi m t)+2 \pi m \sin (2 \pi m t)}{4 \pi^{2} m^{2}+16 k^{3 / 2}} .
$$

If we substitute the form of $f(t)=k_{c}-k+k_{s} f_{s}(t)+k_{a} f_{a}(t)$ into Eq. (10), we obtain

$$
f_{m}=k_{s} \frac{\sin \left(2 \pi m x_{1}\right)}{\pi m}+\frac{k_{a}}{2}\left[\frac{\sin \left(2 \pi(m+1) x_{2}\right)}{\pi(m+1)}+\frac{\sin \left(2 \pi(m-1) x_{2}\right)}{\pi(m-1)}\right],
$$

when $m \neq 0$. With $x_{2}=1 / 4$,

$$
f_{m}=k_{s} \frac{\sin \left(2 \pi m x_{1}\right)}{\pi m}-\frac{k_{a}}{\pi\left(m^{2}-1\right)} \cos \left(m \frac{\pi}{2}\right) .
$$

On the other hand, independently of the form of $f_{m}$, if the convergence time $\Delta t=\theta_{\text {eq }}{ }^{-3}$ is large, we have that $4 k^{3 / 4} \ll 2 \pi$, so we can neglect in Eq. (11) the terms with $k$ and write the crude approximation

$$
\theta_{1}(t)=2 \sum_{m=1}^{\infty} f_{m} \frac{\sin (2 \pi m t)}{2 \pi m}
$$

This approximation is equivalent to neglecting in the right-hand side of Eq. (7) the second term with respect to the first one, that is to say, it implies that $\theta_{1}(t)$ follows $f(t)$ with no delay. We can use it to provide an estimation of the amplitude of the oscillations, with the following simple method. This estimation can be useful in the conceptual design of the satellite.

The largest variations of the slope of $\theta(t)$ and of its approximation $\theta_{1}(t)$ take place at the discontinuities of $f(t)$, namely, at $t=x_{1}, 1-x_{1}$ (in the first period). $f(t)$ changes sign at those times and the periodic function $\theta_{1}(t)$ has its maximum and minimum there. In between, we can take $f(t)$ constant and, therefore, $\theta_{1}(t)$ linear. Hence, we compute the slopes at two convenient points, namely, $t=0,1 / 2$, where $\theta_{1}(t)=0$, according to Eq. (12). We use this information to calculate the maximum and minimum of $\theta_{1}(t)$. The slope at $t=0$ is $f(0)=\left(1-2 x_{1}\right) k_{s}+(1-1 / \pi) k_{a}$, so the maximum is

$$
\theta_{1 \max }=x_{1} f(0)=x_{1}\left[\left(1-2 x_{1}\right) k_{s}+(1-1 / \pi) k_{a}\right] \text {. }
$$

Then, with $k_{s}=0.13, k_{a}=0.007$ and $k_{c}=0.016$ (values to be justified in Sect. 3.3), $\theta_{1 \max }\left(x_{1}=0.4\right)=0.4 \times 0.031=0.012$. The slope at $t=1 / 2$ is $f(1 / 2)=-2 x_{1} k_{s}-k_{a} / \pi$, so the maximum is

$$
\theta_{1 \max }=-\left(1 / 2-x_{1}\right) f(1 / 2)=\left(1 / 2-x_{1}\right)\left[2 x_{1} k_{s}+k_{a} / \pi\right] .
$$

In particular, $\theta_{1 \max }\left(x_{1}=0.4\right)=(0.5-0.4) \times 0.106=0.0106$. Both values agree sufficiently. Since $\theta_{1}(t)$ in Eq. (12) is an odd function, the minimum is at $t=1-x_{1}=0.6$ and its value 
is the negative of the maximum. A graphical comparison of the present approximation with the actual limit cycle is displayed in Fig. 3.

Note that Eqs. (13) and (14) coincide if $k_{a}=0$. In fact, the preceding method relies on the particular form of $f(t)$, namely, it is close to a step function. This is the reason why the method yields a good result. Of course, while still using the approximation leading to Eq. (12), the preceding method is improved by the formula

$$
\theta_{1}(t)=\int_{0}^{t} f(\tau) d \tau
$$

which demands a little more work to yield the maximum value of $\theta_{1}$. Better approximations to $\theta_{1}$ are possible by considering $k$ in Eq. (11), that is to say, by considering the displacement of $\theta_{1}(t)\left[\theta_{1}(0) \neq 0\right]$. In a different sense, the approximation is also improved by carrying on the perturbation scheme to the second order.

\subsubsection{Second order approximation}

Eq. (8) is similar to Eq. (7), if we consider $\theta_{0}=\theta_{\text {eq }}$ and (the square of) the first order solution as the forcing term. Therefore, a similar reasoning leads us to the existence of a periodic solution for $\theta_{2}(t)$ in the large- $t$ limit, which can be obtained by Fourier analysis. The result is

$$
\theta_{2}(t)=-6 k^{1 / 2} \sum_{p=-\infty}^{\infty} \frac{e^{2 \pi i p t}}{2 \pi i p+4 k^{3 / 4}} \sum_{m=-\infty}^{\infty} \frac{f_{m} f_{p-m}}{2 \pi i p-4 \pi^{2} m(p-m)+16 k^{3 / 2}} .
$$

We can see that the perturbation series is a power series in the Fourier coefficients of the forcing term and its convergence depends on the magnitude of this term. Given that this term is proportional to $k_{s}$ and $k_{a}$, the convergence of the series is improved when these constants are small. This condition is related to the one that we found for Eq. (2) to hold as an average equation, namely, that $k=k_{c}+2 x_{1} k_{s}+k_{a} / \pi$ is small. Thus, it is consistent with Eq. (2) as the basis of the perturbation scheme.

\subsection{Numerical solutions}

We use a standard numerical method to integrate Eq. (1), for given values of $k_{s}, k_{a}, k_{c}$. Let us see what values are adequate for a small satellite that is orbiting round the Earth and which can be considered isothermal.

We can take the satellite to be a cube of $0.5 \mathrm{~m}$ side and mass of $50 \mathrm{~kg}$. Let us assume that it is mostly covered by solar cells. Then, suitable values for the absorptivity and emissivity are $\alpha=0.8$ and $\epsilon=0.7$, respectively. We assume the satellite's average specific thermal capacity to be $0.2 \mathrm{~W} \mathrm{~h} \mathrm{~kg}^{-1} \mathrm{~K}^{-1}$ (corresponding to a composition of alluminium with some plastic). An adequate value for the orbital frequency is $(1.5 \mathrm{~h})^{-1}$. Therefore, $C \nu=50 \mathrm{~kg} 0.2 \mathrm{Wh} \mathrm{kg}^{-1} \mathrm{~K}^{-1}(1.5 \mathrm{~h})^{-1}=6.67 \mathrm{~W} / \mathrm{K}$ and $a=A \epsilon \sigma /(C \nu)=$ $1.5 \mathrm{~m}^{2} 0.75 .710^{-8} \mathrm{~W} \mathrm{~m}^{-2} \mathrm{~K}^{-4} /(6.67 \mathrm{~W} / \mathrm{K})=8.9910^{-9} \mathrm{~K}^{-3}$. 


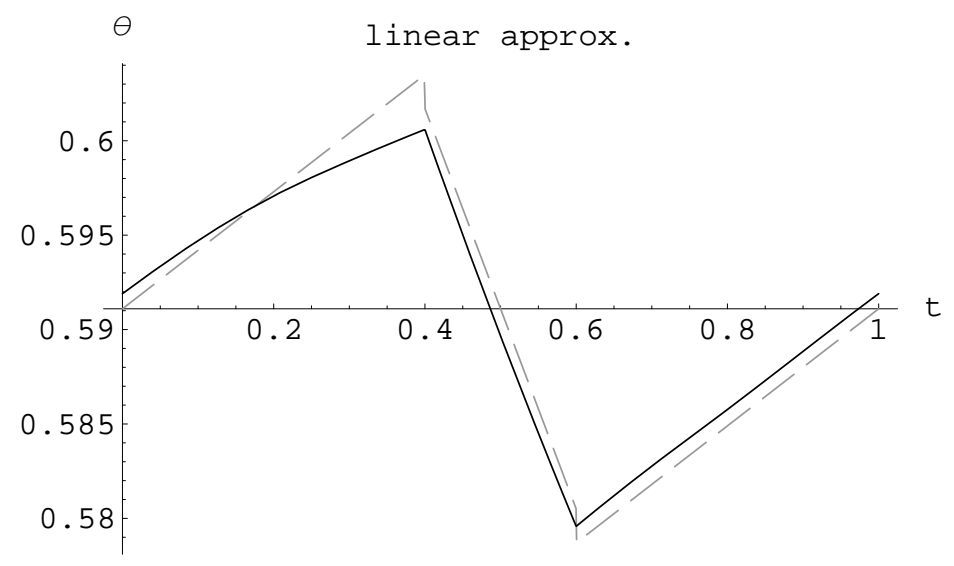

Figure 3: Linear approximation to the first-order perturbative limit cycle $\theta_{\mathrm{eq}}+\theta_{1}(t)$, such that it is linear by pieces (dashed line), compared to the real limit cycle (full line).

The solar irradiation heat input is the product of the solar constant, the absorptivity and the projected area (a quarter of the real area); namely, $\dot{Q}_{s}=1370 \mathrm{~W} \mathrm{~m}^{-2} 0.8\left[6(0.5 \mathrm{~m})^{2} / 4\right]=$ $411 \mathrm{~W}$. Therefore, $k_{s}=a^{1 / 3} \dot{Q}_{s} /(C \nu)=0.128$. The albedo is very variable, and to calculate $\dot{Q}_{a}$, it is necessary, in addition, to consider the visibility factor. We take an average value of $\dot{Q}_{a}=0.05 \dot{Q}_{s}$ so $k_{a}=0.0065$.

The constant heat input is the sum of the equipment dissipation plus the planetary IR irradiation input. The equipment dissipation power is due to the transformation of the incoming irradiation power. If we assume that the solar cells cover $80 \%$ of the satellite's surface and their efficiency is about $10 \%$, and we take into account that the solar irradiation only holds for a fraction $2 x_{1}=4 / 5$ of the period, we deduce that the power dissipated is about $30 \mathrm{~W}$. The planetary IR irradiation input can be estimated by applying the energy balance to the planet (Earth). This balance yields an IR radiation flux of about $7 \%$ of the solar constant. We can take the absorptivity in the IR to be similar to the emissivity $\epsilon=0.7$. Hence, the planetary IR irradiation is about $23 \mathrm{~W}$ and $\dot{Q}_{c}=53 \mathrm{~W}$.

Summarizing, we can take $k_{s}=0.13, k_{a}=0.0070$ and $k_{c}=0.016$. Furthermore, we consider seven values of the initial condition $T(0)$ ( $t=0$ is the noon) uniformly distributed in the interval $273 \mathrm{~K} \leq T \leq 300 \mathrm{~K}$, corresponding to nondimensional $0.57 \leq \theta \leq 0.63$. These values are included between $\theta_{\min }=k_{c}{ }^{1 / 4}=0.36$ and $\theta_{\max }=\left(k_{s}+k_{a}+k_{c}\right)^{1 / 4}=$ 0.625. The results are plotted in Fig. 1 , for the time interval $0 \leq t \leq 5$, which is sufficient to show convergence to the limit cycle.

Indeed, in our example, we are in a situation in which $\theta_{\mathrm{eq}}=\left(k_{c}+4 k_{s} / 5+k_{a} / \pi\right)^{1 / 4}=$ 0.591 is sufficiently small to apply the averaged equation (2). The convergence time is $\Delta t=\theta_{\mathrm{eq}}{ }^{-3}=4.8$. Fig. 1 clearly shows that the evolution is given by an oscillation with unit period superimposed to a slower and approximately exponential convergence in a time $\Delta t$.

To measure precisely the attractor characteristics, we choose the initial condition $\theta(0)=$ $\theta_{\text {eq }}=0.591$ and we let the numerical integration up to $t=10$. The resulting solution has 
$\theta(10)=0.592$, which is slightly different from $\theta_{\text {eq }}$ (although the difference is inappreciable with two-digit precision). Of course, the solution with $\theta(0)=0.592$ is the limit cycle. It has local minimum and maximum amplitudes 0.58 and 0.60 at $t=2 / 5$ and $t=3 / 5$, respectively. Those extreme values are quite close, considering the interval defined by $\theta_{\min }=0.36$ and $\theta_{\max }=0.625$ (see Fig. 1). The limit cycle is plotted in Fig. 3.

\section{Discussion}

Regarding the problem of heat transfer in a satellite, the main conclusions that we can draw from our analysis are the following. First of all, as regards the temperature stability, it holds in the sense of convergence to the limit cycle behaviour, guaranteed by the application of the Poincaré-Bendixson theorem and Dulac's criterion for an annular region. Moreover, the convergence is exponential.

The limit cycle behaviour has a time dependence related to the periodic heat input, namely, approximately similar to its integral, but somewhat displaced. To be precise, the limit cycle consists, in one period, of a temperature-growing phase when the solar heat input is on (as is the albedo), and a relaxation phase in which the temperature falls to adjust to the constant input $\dot{Q}_{c}$ (although this phase ends long before the temperature approaches the corresponding equilibrium value). The total amplitude of the temperature oscillation is relatively small in the example that we have studied. This example, with realistic values of the non-dimensional constants $k_{s}, k_{a}, k_{c}$ for a low-orbit small satellite (reasonably small values, in particular), shows that the attractor is approached exponentially but in a time reasonably larger than the period. This time is simply given in terms of $k_{s}, k_{a}, k_{c}$ by $\Delta t=k^{-3 / 4}$, where $k=k_{c}+2 x_{1} k_{s}+k_{a} / \pi$. Moreover, the mean temperature in the limit cycle is $\theta_{\text {eq }}=k^{1 / 4}$.

It is useful to compare the mean temperature $\theta_{\text {eq }}$ with the oscillation about it. The former depends on $k$, namely, the average of $k_{c}+k_{s} f_{s}(t)+k_{a} f_{a}(t)$, whereas the latter depends on $f(t)$, which is the oscillation of $k_{s} f_{s}(t)+k_{a} f_{a}(t)$ ( $k_{c}$ is a constant). Thus, the temperature oscillation is independent of $k_{c}$ (the constant heat input). This constant is bound to be quite smaller than $k_{s}$, for physical reasons, since the equipment dissipation power is due to the transformation of the incoming solar irradiation power, and the IR irradiation input is a fraction of the solar input. On the other hand, $k_{a} \ll k_{s}$, and we assume that $x_{1}>1 / 4$. In conclusion, the main contributions to $k$ and, therefore, to $\theta_{\mathrm{eq}}$ are due to $k_{s}$. If the temperature oscillation is estimated by Eqs. (13) or (14), the main contribution to it also seems to be due to $k_{s}$; except for the factor $1-2 x_{1}$, which can be small. Let us first note that Eq. (13) yields a larger value than Eq. (14) if $x_{1}>1 /(2 \pi)$, which always holds. Focusing on Eq. (13), we note that the coefficient $1-2 x_{1}$ of $k_{s}$ is smaller than the coefficient of $k_{a}$. When $x_{1}$ approaches $1 / 2$, we only have the $k_{a}$-term: then the satellite is always under the solar irradiation, which does not oscillate; in consequence, the temperature oscillation is only due to the albedo and, therefore, is depressed. 
Let us express the temperature oscillation given by Eq. (13) in physical variables:

$$
\left[T-T_{\mathrm{eq}}\right]_{\max }=a^{-1 / 3} \theta_{1 \max }=x_{1} \frac{\left(1-2 x_{1}\right) \dot{Q}_{s}+(1-1 / \pi) \dot{Q}_{a}}{C \nu} .
$$

Contrary to the expression of $T_{\text {eq }}$ in Eq. (4), this expression depends on $C$ and $\nu$ but it does not depend on $A, \epsilon$ and $\sigma$. We expect both expressions to be very useful for conceptual thermal design.

Of course, in the numerical example in Sect. 3.3 we have not considered every orbital circumstance and we have just intended to find a set of sensible values for the constants. More information on satellite design and the space thermal environment can be found in the literature [1]. It is worthwhile to discuss briefly here possible changes of the values of $k_{s}$ and $k_{c}$. While the ratio $k_{c} / k_{s} \simeq 0.1$ is adequate for a small satellite, the absolute values of $k_{s}, k_{c}$ can be amply changed; for example, by increasing the period (which we have taken as $1.5 \mathrm{~h}$ in our case). It is easy to see that both $k_{s}$ and $k_{c}$ are proportional to the $4 / 3$ rd-power of the period. Thus, if we increase the period by a factor of ten, say, the constants increase by a factor of $10^{4 / 3}$, so that $\Delta t=\left(k_{c}+2 x_{1} k_{s}+k_{a} / \pi\right)^{-3 / 4}$ decreases by a factor of ten, becoming smaller than one (the period). In this situation, the convergence to the limit cycle is very fast. In fact, it is appropriate to consider the opposite approximation to the temperature evolution, namely, during the decay to the limit cycle, the temperature only changes slightly along this limit cycle.

Further analysis could be based on a more refined model than the isothermal model that we have used. In particular, a two-node model has been studied by Guerra, Pérez-Grande and Sanz [7]. The two ODE's for the two-node model are equivalent to an autonomous system of three ODE's. As is well known, such system can have chaotic behaviour [4]. However, we expect that the situations that are well approximated by the one-node model that we have analysed do not exhibit chaotic features and, therefore, can be conveniently studied, and provide, in addition, a basis for this very simple model.

\section{Acknowledgments}

We thank Antonio Barrero-Gil for conversations.

\section{References}

[1] P. Fortescue and J. Stark (eds.), Spacecraft Systems Enginnering (Wiley, Chichester, 1995); G. Gilmore (ed.), Spacecraft Systems Control Handbook (The Aerospace Press, El Segundo, 2002)

[2] M.W. Hirsch and S. Smale, Differential Equations, Dynamical Systems, and Linear Algebra, Pure and Applied Mathematics (Academic Press, N.Y., 1974)

[3] A.A. Andronov, A.A. Vitt and S.E. Khaikin, Theory of Oscillators, (Dover, N.Y., 1987) 
[4] P.G. Drazin, Nonlinear Systems, Cambridge texts in applied mathematics (Cambridge U.P., 1992)

[5] V.I. Arnold, Ordinary Differential Equations (MIT Press, Cambridge, 1973)

[6] C.M. Bender and S.A. Orszag, Advanced Mathematical Methods for Scientists and Engineers (Springer, N.Y., 1999)

[7] C. Guerra, I. Pérez-Grande and A. Sanz-Andrés, Analytical study of the thermal behaviour of a small satellite, submitted to Applied Thermal Engineering. 